\title{
Potential use of azithromycin alone and in combination with ivermectin in fighting against the symptoms of COVID-19.
}

1. MBBS, M.Phil, P.hD Associate Professor Pharmacology Rehman Medical College, Peshawar. 2. MBBS, M.Phil

Medical Officer

Govt. Sifat Ghayur Shaheed Memorial Hospital, Peshawar.

3. MBBS, M.Phil

Associate Professor Pharmacology Sheikh Zayed Medical College, Rahim Yar Khan.

Correspondence Address: Dr. Rizwan Faisal

Department of Pharmacology Rehman Medical College, Peshawar. rizwan.faisal@rmi.edu.pk

Article received on:

03/08/2020

Accepted for publication

$15 / 10 / 2020$

\section{Rizwan Faisal', Syed Furqan Ali Shah ${ }^{2}$, Mazhar Hussain ${ }^{3}$}

ABSTRACT... Objectives: To compare efficacy of azithromycin alone and in combination with ivermectin against the duration of novel corona virus symptoms. Study Design: Cross-sectional study. Setting: Shah Care Hospital, Peshawar. Period: April 5, 2020 to May 30, 2020. Material \& Methods: Total patients included in the study were 100. Patients were divided into two groups by systematic random sampling: Group A: who received AZM (500mg once a day for 5 days), Group B: who received Ivermectin (12mg once a day for 5 days) and AZM (500mg once a day for 5 days). All the participants were informed to revisit hospital as soon as symptoms (at least two of the mentioned symptoms) like fever, fatigue, sore throat, cough, body aches/myalgia, anosmia/hyposmia, ageusia/hypogeusia and diarrhea disappears at least for 3 days (the actual day on which symptom(s) disappeared was noted). They were also advised to revisit hospital if they feel any inconvenience with the treatment or they notice worsening of the symptoms. The efficacy of the two regimens was based on the duration of disappearance of symptoms. RT-PCR was repeated after 15 days of the diagnosis and on day 21 who came positive on day 15. Results: In group A, the symptoms of $34 \%$ patients started to disappear during $3-5$ days (mean \pm SD, $4.86 \pm 0.42$ days) following therapy, it disappeared during 6-8 days $(7.18 \pm 0.37$ ) in $28 \%$ patients, $22 \%$ were symptom free during $9-10$ days $(10.12 \pm 0.12)$, and $16 \%$ took $>10$ days to become symptom free $(12 \pm 0.26)$. Similarly, $52 \%$ of group B patient were relieved during 3-5 days (4.01 \pm 0.32$)$ of therapy, $36 \%$ were symptom free during $6-8$ days $(6.32 \pm 0.14)$, symptoms of $8 \%$ disappeared during 9 -10days $(9.06 \pm 0.25)$, and $4 \%$ took $>10$ days $(11 \pm 0.0)$ to become symptom free. Conclusion: Combination of ivermectin and azithromycin was more effective in making patients symptom free than azithromycin alone.

Key words: $\quad$ Azithromycin, Coronavirus, Ivermectin, Severe Acute Respiratory Syndrome.

Article Citation: Faisal R, Shah SFA, Hussain M. Potential use of azithromycin alone and in combination with ivermectin in fighting against the symptoms of COVID-19. Professional Med J 2021; 28(5):737-741.

https://doi.org/10.29309/TPMJ/2021.28.05.5867

\section{INTRODUCTION}

Ivermectin is broad spectrum, FDA-approved anti-parasitic agent. ${ }^{1}$ It has been found to possess anti-viral activity against a wide range of viruses. Several studies have been conducted in vitro in order to prove its efficacy and potency. ${ }^{2,3,4,5}$ Since decades many infectious diseases are being treated with this magical drug. Many studies have showed less adverse effects associated with the oral use of ivermectin. This magical drug was discovered in late 1970s, it was approved in 1981 to be used in animals. After a few years it was also used in animals. William C. Campbell and Satoshi Ōmura who discovered it were given Nobel Prize in 2015. $6,7,8$
It is lipid soluble drug which adds more to its beauty, and hence plays very vital role in treating many parasitic as well as viral infections through a variety of mechanisms. ${ }^{6}$ Moreover, immunomodulation in the host were also reported by this drug. Researches revealed its actions on hindering the proliferation not only on cancer cell but also affect cholesterol and glucose regulation in animals. This drug exerts multiple effects but many of its underlying mechanisms are still not known. ${ }^{9}$

The severe acute respiratory syndrome coronavirus 2 (SARS-CoV-2) leads to severe acute respiratory syndrome. It is a singlestranded RNA virus. It was initially called SARS- 
CoV-2 but later on named as COVID-19 by World Health Organization officially. First identified case of COVID-19 was known in 2019, December in China, later on spread all over the world while the definite behavior and pathogenicity of the virus are still not completely understood..$^{10,11}$

Ivermectin is extremely useful against many microorganisms including some viruses. However, the guidelines for clinical management have been reviewed frequently and numerous immunomodulatory and antiviral drugs have been recommended. Due to outbreak of COVID-19 many herbal agents were also being used for the cure but still it needs lots of studies to clarify the role and outcome in this deadly virus. Hundreds of clinical trials are presently in progress. ${ }^{12,13}$

As morbidity and mortality associated with COVID-19 is high and no definite drug treatment against it is available, therefore at this current point of time, it is a major need to find the likely effective drugs which either have the potential to treat the coronavirus infected person or to stop the progression of disease towards complications. Ivermectin performs a vital part in numerous biological processes and showed effectiveness against several viruses, hence it may work as a possible alternative in the cure of COVID-19 as well. The current study is an attempt to investigate the efficacy of ivermectin against the COVID-19. The result of the study if revealed positive response will provide a good insight to the treatment of COVID-19.

\section{MATERIAL \& METHODS}

This cross-sectional study was conducted in Shah Health Center, Peshawar from April 5, 2020 to May 30, 2020 after ethical approval. All patients of COVID-19 who visited the center during study period were included in the study after confirmation by RT-PCR. Complete blood count (CBC), electrocardiography (ECG), c-reactive proteins (CRP) and chest x-ray were performed before starting the treatment. Patients with associated severe co-morbidities like diabetes mellitus, cardiovascular problems, chronic renal failure and oxygen dependent patients were excluded from the study. After detailed history, proper examination and investigations were done, written informed consent was taken after explaining study to the patients. Their confidentiality was maintained. ${ }^{14,15}$ Study comprised of 100 patients which were distributed randomly into two groups: Group A: who received AZM (500mg once a day for 5 days), Group B: who received Ivermectin (12mg once a day for 5 days) and AZM (500mg once a day for 5 days). Both the groups also received tablets of paracetamol $(500 \mathrm{mg}$ if needed), Vit C (500mg once a day for 15 days), zinc (20mg twice a day for 15 days) and Vit $D$ (injection PO 200,000units once) supplements. All the patients were instructed to revisit hospital as shortly as symptoms (at least two of the mentioned symptoms) like fever, fatigue, sore throat, cough, body aches/myalgia, anosmia/ hyposmia, ageusia/hypogeusia and diarrhea disappears at least for 3 days (the actual day on which symptom(s) disappeared was noted). They were also advised to revisit hospital if they feel any inconvenience with the treatment or they notice worsening of the symptoms. The efficacy of the two regimens was based on the duration of disappearance of symptoms. CBC, ECG, CRP and chest x-ray of all the patients were repeated after one week of starting the therapy. RT-PCR was repeated after 15 days of the diagnosis, 73 cases were found negative in first PCR while 27 were still positive. After 1 week RT-PCR was again repeated for positive cases and this time it was negative for all.

Data was analysed by using SPSS16 version. Quantitative variables like age of patients and duration of symptoms were described as mean and standard deviation (SD). Data among groups was analysed by using student t-test. p-value $\leq$ 0.05 was considered statistically significant.

\section{RESULTS}

This study included 100 patients (50 in each group), the efficacy of the two regimens was compared on the duration of disappearance of symptoms. Female participants were 20 while male were 80 . Age distribution among groups was analyzed as in Group A mean age was $46 \pm 2.13$ years, mean age in Group B was $46 \pm 3.42$ years. Regarding efficacy, the symptoms of $34 \%$ patients 
of group A started to disappear during 3-5 days following therapy, it disappeared during 6-8 days in $28 \%$ patients, $22 \%$ were symptom free during 9-10 days and $16 \%$ patients took more than 10 days. Similarly, $52 \%$ of group B patient shown no symptoms during 3-5 days of therapy, $36 \%$ were symptom free during 6-8 days, $8 \%$ were having no symptoms between day $9-10$ and $4 \%$ took more than 10 days to become symptom free (Table-I). Time taken by mean number of patients against disappearance of symptoms in group $A$ and $B$ are shown in Table-II.

When group A was compared with group B at 3-5 days, 6-8 days, 9-10 days and $>10$ days, a significant difference of $p$-value $<0.01$ was found at each reading. The comparison of duration of different clinical features to disappear is shown in Figure-1.

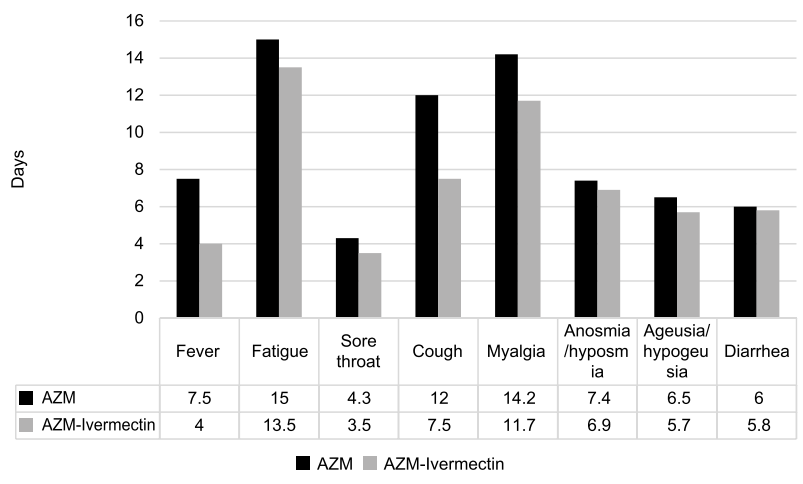

Figure-1. Effect of AZM and AZM-Ivermectin on the duration of COVID-19 symptoms

\begin{tabular}{|l|c|c|c|c|}
\hline \multicolumn{1}{|c|}{ Groups } & $\mathbf{3 - 5}$ Days & $\mathbf{6 - 8}$ Days & $\mathbf{9 - 1 0}$ Days & $\mathbf{> 1 0}$ Days \\
\hline Group A (AZM) & $17(34 \%)$ & $14(28 \%)$ & $11(22 \%)$ & $8(16 \%)$ \\
\hline Group B (AZM-Ivermectin) & $26(52 \%)$ & $18(36 \%)$ & $4(8 \%)$ & $2(4 \%)$ \\
\hline
\end{tabular}

Table-I. Frequency of patients versus time taken for disappearance of COVID-19 symptoms $(n=100)$

\begin{tabular}{|l|c|c|c|c|}
\hline \multicolumn{1}{|c|}{ Groups } & 3-5 Days & $\mathbf{6 - 8}$ Days & $\mathbf{9 - 1 0}$ Days & $>$ 10 Days \\
\hline Group A (AZM) & $4.86 \pm 0.42$ & $7.18 \pm 0.37$ & $10.12 \pm 0.12$ & $12 \pm 0.26$ \\
\hline Group B (AZM-Ivermectin) & $4.47 \pm 0.32$ & $6.32 \pm 0.14$ & $9.06 \pm 0.25$ & $11 \pm 0$ \\
\hline p-value & 0.01 & 0.01 & 0.002 & 0.002 \\
\hline
\end{tabular}

Table-II. Mean \pm SD of patients versus time taken for disappearance of COVID-19 symptoms $(n=100)$

\section{DISCUSSION}

Ivermectin, is a fellow of the avermectin family, produced by the microorganism present in soil, Streptomyces avermitilis. ${ }^{16}$ It has a variety of actions which ranges from endo and/or ectoparasiticide action to anticancer, antiviral and antibacterial actions. ${ }^{17}$

Majority of the patients (49\%) developed multiple symptoms. In solo symptom fever was the most common (16\%) followed by myalgia (13\%). AZMIvermectin combination was able to improve all the symptoms of COVID-19 as compared to AZM alone but has pronounced effects on the duration of fever and myalgias (Figure-1).

A latest in vitro research was performed, exposure of the Vero/hSLAM cells which were infected with the SARS-CoV-2 to ivermectin $(5 \mu \mathrm{M})$ in 48 hours resulted in a decrease of 5000 fold in viral RNA as compared to control. The results revealed killing of almost all the viral particles within $48 \mathrm{~h}$ with ivermectin treatment. The importin (IMP) $\alpha / \beta$ receptor, carries viral proteins to the nucleus of host cell were also inhibited by ivermectin, indicating its antiviral effects. The authors of the study suggested to check the likely effectiveness of ivermectin in COVID-19 patients. Researchers got hope after this discovery that ivermectin can be repurposed for COVID-19 treatment. $^{18}$

It has also been hypothesized that hydroxychloroquine in combination with ivermectin against SARS-CoV-2 may produce a synergistic inhibitory effect. Hydroxychloroquine results in inhibition of the entrance of SARS-CoV-2 into the host cells, while ivermectin promote the antiviral action by impeding replication of virus. ${ }^{19}$ After showing encouraging results by ivermectin in an in vitro study, the efficacy of ivermectin treatment was assessed in an registry-based observational study comprising seriously ill 
patients infected with SARS-CoV-2. Treatment with $150 \mu \mathrm{g} / \mathrm{kg}$ ivermectin was found to decrease mortality rate and use of healthcare resources. ${ }^{6}$ Results of this initial study gave confidence for the usage of ivermectin in clinical setup. ${ }^{20}$

\section{CONCLUSION}

Combination of azithromycin \& ivermectin was more effective in relieving patient's symptoms as compare to azithromycin alone.

\section{Limitation of the Study}

As this study is based on a single center, therefore, more studies are required at wide level to generalize the results.

\section{Conflict of Interest}

We, the authors declare that we have no conflict of interest.

\section{Source of Financial Support}

It is not supported financially by any one.

\section{Copyright $\odot 15$ Oct, 2020.}

\section{REFERENCES}

1. Canga AZ, Prieto AMS, Liébana MJD, Martínez NF, Vega MS, and Juan J. García Vieitez JJG. The pharmacokinetics and interactions of ivermectin in humans--a mini-review. AAPS J. 2008:10(1):42-46

2. Götz V, Magar L, Dornfeld D, Giese S, Pohlmann $A$, Höper D et al. Influenza A viruses escape from MxA restriction at the expense of efficient nuclear vRNP import. Sci. Rep. 2016; 6:23138.

3. Lundberg L, Pinkham C, Baer A, Amaya M, Narayanan $A$, Wagstaff $K M$ et al. Nuclear import and export inhibitors alter capsid protein distribution in mammalian cells and reduce Venezuelan Equine Encephalitis Virus replication. Antivir. Res. 2013; 100(3):662-72.

4. Tay MYF, Fraser JE, Chan WKK, Moreland MJ, Rathore AP, Wang $C$ et al. Nuclear localization of dengue virus (DENV) 1-4 non-structural protein 5; protection against all 4 DENV serotypes by the inhibitor Ivermectin Antivir. Res. 2013; 99(3):301-06.

5. Wagstaff KM, Rawlinson SM, Hearps AC, Jans DA. An Alpha Screen(R)-based assay for high-throughput screening for specific inhibitors of nuclear import. J. Biomol. Screen, 2011; 16(2):192-200.
6. Crump A, Ōmura S. Ivermectin, 'wonder drug' from Japan: The human use perspective. Proc Jpn Acad Ser B Phys Biol Sci. 2011; 87:13-28.

7. Kircik LH, Del Rosso JQ, Layton AM, Schauber J. Over 25 years of clinical experience with ivermectin: An overview of safety for an increasing number of indications. J Drugs Dermatol. 2016; 15:325-32.

8. Voorhis WCV, Huijsduijnen RHV, Wells T. Profile of William C. Campbell, Satoshi Ōmura, and Youyou Tu, 2015 Nobel Laureates in Physiology or Medicine. Proc Natl Acad Sci 2015; 112(52):67-72.

9. Laing R, Gillan V, Devaney E. Ivermectin-old drug, new tricks? Trends Parasitol. 2017; 33:463-72.

10. Sohrabi C, Alsafi Z, O'Neill N et al. World Health Organization declares global emergency: A review of the 2019 novel coronavirus (COVID-19). Int J Surg. 2020; 76:71-6.

11. Lu H, Stratton CW, Tang Y-W. Outbreak of pneumonia of unknown etiology in Wuhan, China: The mystery and the miracle. J Med Virol. 2020; 92:401-2.

12. Lu CC, Chen MY, Chang YL. Potential therapeutic agents against COVID-19: What we know so far. J Chin Med Assoc. 2020; 10.1097/JCMA.0000000000000318.

13. Ang L, Lee HW, Choi JY, Zhang J, Soo Lee M. Herbal medicine and pattern identification for treating COVID-19: A rapid review of guidelines. Integr Med Res. 2020; 9:100407.

14. Faisal R, Rehman K, Bahadur S, Shinwari L. Problem based learning in comparison with lecture based learning among medical students. Journal of Pakistan Medical Association. 2015; 66(6):650-3.

15. Faisal R, Shinwari L, Izzat S. Academic performance of day scholars versus boarders in pharmacology examinations of a medical school in Pakistan. J Pak Med Assoc. 2016; 66(9):1094-97.

16. Campbell WC, Benz GW. Ivermectin: a review of efficacy and safety. J Vet Pharmacol Ther. 1984; 7(1):116.

17. Crump A, Omura S. Ivermectin, 'wonderdrug' from Japan: The human use perspective. Proceed Japan Acad Series B. 2011; 87(2):13-28.

18. Caly L, Druce JD, Catton MG, Jans DA, Wagstaff KM. The FDA-approved drug ivermectin inhibits the replication of SARS-CoV-2 in vitro. Antiviral Res. 2020:104787. 
19. Patrì $A$, Fabbrocini G. Hydroxychloroquine and ivermectin: A synergisticcombination for COVID-19 chemoprophylaxis and/or treatment? J Am Acad Dermatol. 2020; 82(6):e221.
20. Millan-Oñate J, Millan W, Mendoza LA, Sánchez CG, Fernandez-Suarez H, Bonilla-Aldana DK, RodriguezMorales AJ. Successful recovery of COVID-19 pneumonia in a patient from Colombia after receiving chloroquine and clarithromycin. Ann Clin Microbiol Antimicrob. 2020; 12941-020-00358-y.

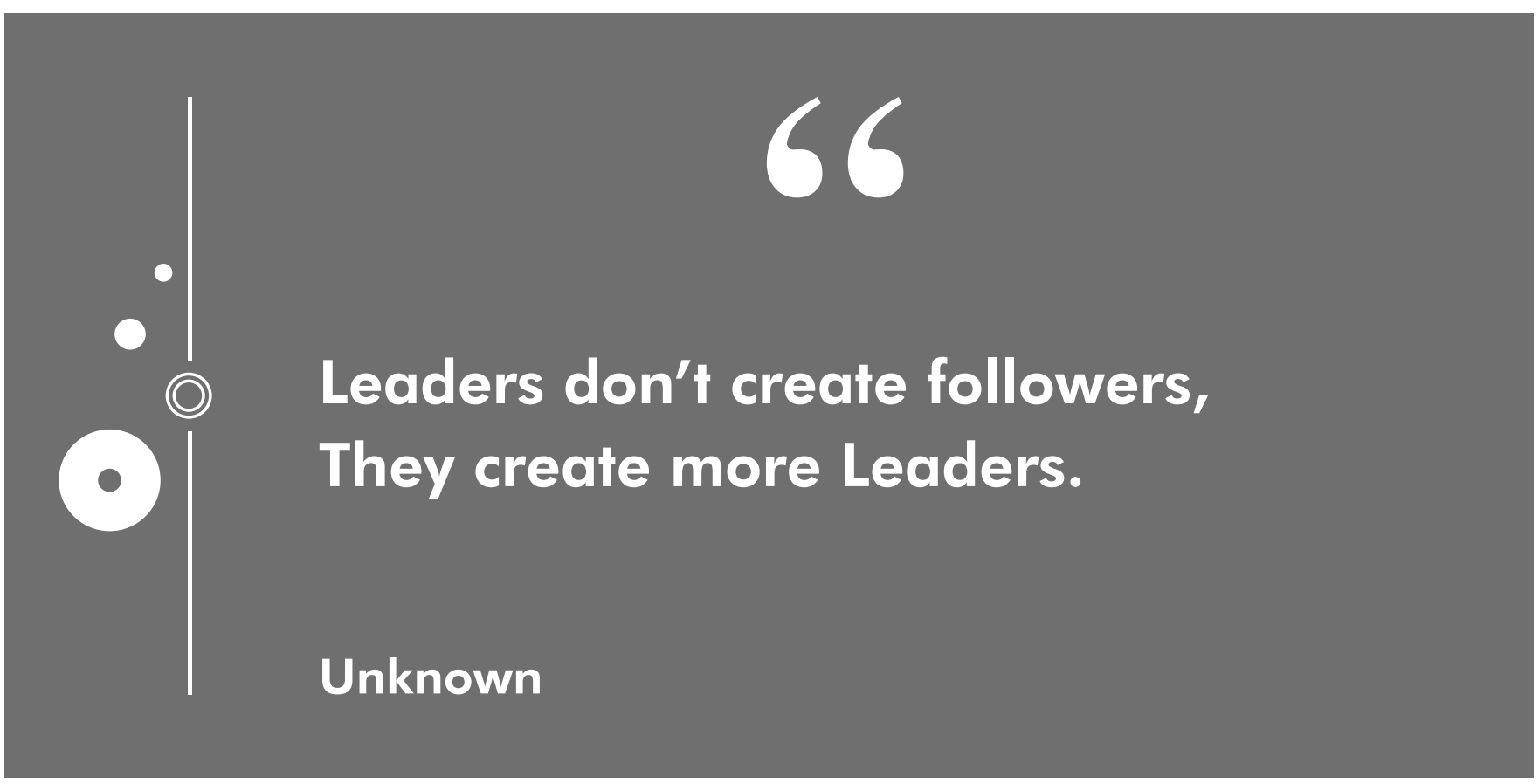

\section{AUTHORSHIP AND CONTRIBUTION DECLARATION}

\begin{tabular}{|c|l|l|l|}
\hline Sr. \# & Author(s) Full Name & \multicolumn{1}{|c|}{ Contribution to the paper } \\
\hline 1 & Rizwan Faisal & $\begin{array}{l}\text { Conception \& design, acquisition } \\
\text { of data, analysis \& interpretation } \\
\text { of data, Drafting the article. } \\
\text { Design of study, acquisition of } \\
\text { data, analysis \& interpretation of } \\
\text { data, drafting. } \\
\text { Design of study, acquisition of } \\
\text { data, analysis \& interpretation of } \\
\text { data, Drafting. }\end{array}$ \\
\hline 3 & Syed Furqan Ali Shah \\
\hline
\end{tabular}

